\section{P184 Promoting Healthful Beverage Choices among Households Enrolled in the Supplemental Nutrition Assistance Program (SNAP)}

Jamie Benedict,PhD,LDN, RD, jamieb@cabnr.unr.edu, University of Nevada, Reno, Department of Agriculture, Nutrition and Veterinary Sciences, Reno, NV 89557; Chenin Treftz, PhD, RDN, University of Nevada, Reno; Deborah Joakimson, BS, DTR; Elizabeth Christiansen, PhD

Objective: To implement a multi-component campaign that promotes healthful beverage choices and reduces the intake of sugary drinks.

Theory, Prior Research, Rationale: The goal of this program is to increase the likelihood that parents will monitor and limit their children's intake of sugary drinks. To achieve this goal, a multi-component campaign was developed based on the principles of social marketing and the social-ecological theory.

Description: Rethink Your Drink Nevada includes a direct-mail intervention to eligible SNAP households; provision of patient educational materials to primary health care providers and dentists; and a print media campaign. Digital resources include a newsletter, website and Facebook page. Target audience was SNAP households with one or more children between the ages of 6 and 12 .

Evaluation: The findings presented here focus on the mail survey results of 1,000 randomly-selected SNAP households regarding the direct-mail intervention conducted in Washoe County, NV. Among parents who had reportedly read "some or nearly everything" of the materials $(66 \%)$, most found them interesting to read $(87 \%)$, indicated they had learned something new (69\%), and had used one or more of the suggested strategies (94\%). They were also more likely to monitor and limit their children's intake of sugary drinks compared to those parents' who did not recall receiving the materials or read none or very little of them $(\mathrm{p}<.05)$.

Conclusions and Implications: These findings suggest that a direct-mail intervention may be effective in changing parents' behaviors that serve to reduce children's intake of sugary drinks. In addition, sending educational materials via the United States Postal Service remains a viable option to reach SNAP households with nutrition information.

Funding: Supplemental Nutrition Assistance Program Education

\section{P185 PSE Approaches to Expand Reach in SNAP-Ed Programming}

Kendra Auguste, MA, MPH, kauguste@ufl.edu, University of Florida, 1408 Sabal Palm Drive, 2nd Floor, Gainesville, FL 32611; Elder Garcia, MA, MPA, University of Florida; Lauren Headrick, MS, RD; Karla Shelnutt, PhD, RD

Objective: To identify opportunities to expand policy, system and environmental (PSE) approaches to strengthen SNAP-Ed programming in Florida and increase the number of participants adopting healthier behaviors.

Rationale: By implementing PSEs in low-resource communities, residents have an increased opportunity to adopt and sustain nutrition and physical activity habits.

Description: Expanding from 37 to 51 Florida counties, the University of Florida/IFAS Extension Family Nutrition Program (FNP) (Florida's SNAP-Ed program) reached $64 \%$ more SNAP-Ed participants overall through direct nutrition education and PSE changes in FY16. By focusing on specific settings, FNP increased collaboration with local partners and stakeholders and expanded its PSE change efforts. In order to accomplish expansion, FNP mobilized two specialized teams, Farm to School and Community and Public Health, to implement strategies in each of these areas. Target audience was SNAP-eligible residents in communities across the state of Florida.

Evaluation: In FY15, FNP reported 95 partners throughout the state, which increased to 942 partners in FY16. FNP also implemented a total of 183 PSE approaches in FY16, reaching 310\% more Floridians than in FY15. These efforts were tracked through monthly reporting on specific indicators from the SNAP-Ed evaluation framework. PSE approaches included implementing community and school gardens, engaging school wellness committees, participating in community health coalitions, and improving access to fresh produce.

Conclusions and Implications: Identifying opportunities to support nutrition education at existing sites and collaborating with community partners in new settings can strengthen SNAP-Ed programming within communities.

Funding: Supplemental Nutrition Assistance Program Education

\section{P186 SNAP Benefits Go Beyond Food Access: The Impact on Cost-Related Medication Nonadherence for Older Adults}

Mithuna Srinivasan, PhD, mithunasrinivasan@gmail.com, IMPAQ International, LLC, 1101 Vermont Avenue NW, Washington, DC 20005; Jennifer A. Pooler, MPP, IMPAQ International, LLC

Objective: One in five older adults engage in costrelated medication nonadherence (CRN), which can result in hospitalizations and adverse health outcomes, and rates are higher for those at-risk of hunger. This study sought to examine whether participation in the Supplemental Nutrition Assistance Program (SNAP) may help older adults better afford their medications, plausibly by reducing out-of-pocket food expenditure.

Study Design, Setting, Participants, Intervention: Using data from the 2013-2015 U.S. National Health

Continued on page 597 